\title{
Propiedades Volumétricas de la DL-Alanina en Soluciones Acuosas del Líquido lónico Cloruro de 1-Butil-3- Metilimidazolio a las Temperaturas desde 283.15 hasta 313.15 K.
}

\author{
Manuel S. Páez ${ }^{(1) \star}$, María K. Vergara ${ }^{(1)}$ y Omar A. Pérez ${ }^{(2)}$ \\ (1) Universidad de Córdoba. Departamento de Química. Facultad de Ciencias Básicas. Carrera 6 No. 76 - \\ 103. Km 3.Vía Cereté. Córdoba. Colombia. e-mail: (mspaezm@unal.edu.co) \\ (2) Universidad de Córdoba. Departamento de Ingeniería de Alimentos. Facultad de Ciencias Agrícolas. \\ Carrera 6 No. 76-103. Km 3. Vía Cereté. Córdoba-Colombia.
}

*Autor a quien debe dirigirse la correspondencia.

Recibido Mar. 24, 2015; Aceptado May. 22, 2015; Versión final Jul. 17, 2015, Publicado Oct. 2015

\begin{abstract}
Resumen
Se determinaron las densidades de soluciones acuosas del aminoácido DL-Alanina + Cloruro de 1-Butil-3Metilimidazolio [Bmim $\left.{ }^{+}\right][\mathrm{Cl}]$, en el rango de concentración 0 hasta $1.0007 \mathrm{~mol}^{\mathrm{kg}} \mathrm{kg}^{-1}$ a temperaturas desde 283.15 hasta $318.15 \mathrm{~K}$ a intervalos de $5 \mathrm{~K}$, utilizando un densímetro de tubo vibratorio Anton Paar DMA 5000 , en el intervalo de solubilidad permitido. Se calcularon los volúmenes molares aparentes a dilución infinita, la segunda derivada del volumen molar parcial a dilución infinita con respecto a la temperatura y el volumen molar parcial de transferencia a dilución infinita. Los valores del volumen molar parcial de transferencia a dilución infinita son negativos. Esto indica que predominan las interacciones entre iones del aminoácido y grupos no polares del líquido iónico, o viceversa También se encontró que la DL-Alanina se comporta como un soluto hidrofílico en las mezclas seudobinarias que contienen al líquido iónico $[\mathrm{Bmim}+]\left[\mathrm{Cl}^{-}\right]$.
\end{abstract}

\section{Volumetric properties of DL-Alanine in Aqueous Solutions of 1-Butyl-3-Methylimidazolium Chloride at Temperatures from 283.15 to $313.15 \mathrm{~K}$}

\begin{abstract}
Densities of aqueous mixtures of DL-alanine + 1-butyl-3-methylimidazolium chloride $\left[\mathrm{Bmim}^{+}\right]\left[\mathrm{Cl}^{-}\right]$were measured over the concentration range 0 to $1.0007 \mathrm{~mol}^{\mathrm{kg}}{ }^{-1}$ at temperatures from 283.15 to $313.15 \mathrm{~K}$ at intervals of $5 \mathrm{~K}$, using an Anton Paar DMA 5000 vibrating tube densitometer, within the allowable range of solubility. The infinite dilution apparent molar volume, the second derivative of the infinite dilution partial molar volume with respect to temperature, and the transfer partial molar volume at infinite dilution were calculated. The values of the transfer partial molar volume at infinite dilution are negative. This indicates the predominant interactions between ions of the amino acid and the non-polar groups of the ionic liquid or vice versa. It also was found that the DL-alanine acts as an hydrophilic solute in the pseudo binary mixtures containing the ionic liquid $\left[\mathrm{Bmim}^{+}\right]\left[\mathrm{Cl}^{-}\right]$.
\end{abstract}

Keywords: volumetric properties, infinite dilution volume, ionic liquids, DL-alanine 


\section{INTRODUCCIÓN}

Los electrolitos orgánicos incluyendo a los líquidos iónicos pueden brindar información acerca del efecto de las interacciones electrostáticas e hidrofóbicas sobre la estabilidad de los aminoácidos en medio acuoso, ya que estos pueden ser hidrofóbicos o hidrofílicos dependiendo de la estructura del catión y/o anión; pudiendo disociar parcial o totalmente en agua, tal como ocurre normalmente con las soluciones acuosas de las sales inorgánicas; durante este proceso los iones son solvatados en solución causando cambios estructurales en el comportamiento de la mezcla (Luciana et al., 2009). Por ello, es de esperar que los líquidos iónicos influyan sobre las conformaciones macromoleculares en sistemas biológicos, a través del debilitamiento de las interacciones repulsivas o atractivas (inter o intra cadena y carga-carga), afectándose de esta forma las interacciones hidrofóbicas de las cadenas laterales de los grupos alquílicos (Shekaari et al., 2010).En adición a lo anterior se sabe que la mayoría de los procesos bioquímicos que implican diversos tipos de interacciones de biomoléculas con fármacos (Rajagopal et al., 2009) e iones ocurren en medios acuosos.

Procesos bioquímicos de gran relevancia, tales como la desnaturalización de una proteína en soluciones acuosas salinas, son objeto de una amplia investigación (Bennion et al., 2003; England et al., 2011; Amani et al., 2011) y aún no son bien comprendidos, tal vez a causa de los complejos aspectos de configuración y conformación que adquieren estos compuestos en soluciones (Constantinescu et al., 2007). Así estos hechos evidencian una vez más la dificultad existente para la comprensión de estos procesos biológicos complejos.

Las soluciones acuosas de aminoácidos mezcladas con líquidos iónicos pueden ser estudiadas con el fin de obtener información sobre las interacciones soluto-solvente y comprender el papel de estas en la estabilidad conformacional de las proteínas, a través de mecanismos moleculares sencillos que podrían ser develados con ayuda del comportamiento fisicoquímico; los cuales no necesariamente deben ser abordados desde el estudio de macromoléculas biológicas en solución, sino usando compuestos modelos más sencillos, tales como los aminoácidos en solventes mixtos.

Es por ello que en la última década, se han realizado diversas investigaciones del estudio termodinámico de sistemas seudobinarios que contienen sustancias electrolíticas (Páez et al., 2015; Yan et al., 2014; Páez F et al., 2014; Shekaari et al., 2010; Qiao et al., 2010; Singh et al., 2007; Dhondge et al., 2012;Jahagirdhar et al., 1998), considerando que las propiedades termofísicas y termodinámicas son útiles para entender las interacciones moleculares (hidrofílicas, hidrofóbicas e iónicas) de diversos solutos en sistemas binarios y seudobinarios. Los líquidos iónicos han sido utilizados como medio novedoso para investigar procesos que contienen biomoléculas (Swatloski et al., 2002; van Rantwijk et al., 2007).

Específicamente, en este estudio se considera que las propiedades volumétricas de aminoácidos en soluciones salinas nos permitiría obtener información acerca de las interacciones presentes. Por tal razón, se decidió estudiar las propiedades volumétricas de la DL-Alanina en soluciones acuosas de cloruro de 1-butil3-metilimidazolio a las temperaturas desde 283.15 hasta $313.15 \mathrm{~K}$, con el objetivo de contribuir a un mejor entendimiento del efecto que tiene este soluto sobre el predominio de las interacciones moleculares que ocurren en solución.

\section{MATERIALES Y MÉTODOS}

Se empleó DL-Alanina con un grado de pureza de 99\% (Alfa Aesar) y el líquido iónico [Bmim+][Cl-] con una pureza $\geq 99 \%$ (Across Organic). La DL-Alanina fue recristalizada a partir de soluciones acuosas de etanol y se secó bajo vacío a $348 \mathrm{~K}$ durante 6 horas, luego se almacenó sobre $\mathrm{P}_{2} \mathrm{O}_{5}$ en un desecador antes de su uso (Wang et al., 2004). Para reducir el contenido de agua y los compuestos volátiles a valores insignificantes de este líquido iónico, se secó con agitación a temperatura moderada $(T=343 \mathrm{~K})$ y bajo vacío $(\mathrm{p}=0,2 \mathrm{~Pa})$ aproximadamente durante 48 horas antes de su uso. Después de secado se guardó en botellas bajo una atmósfera de gas inerte. Luego se midió el contenido de agua de este líquido iónico usando un Coulómetro Karl Fisher (Metrohm 831). En este proceso, el yodo se genera eléctricamente en la solución de Karl Fisher y el contenido de agua se determina por la cantidad de yodo que reacciona con el agua en una relación de 1:1. Para este propósito se usó un tamaño de muestra de aproximadamente 0,1 gramo en cada medición, y se repitió dos veces para cada muestra, dando como resultado un contenido de agua de 200 ppm (Páez et al., 2014). El agua usada para preparar las soluciones fue bidestilada en medio alcalino y $\mathrm{KMnO}_{4}$ para eliminar cualquier materia orgánica y posteriormente desgasificada presentando una conductividad inferior a $2 \mu \mathrm{S} . \mathrm{cm}$ 1.

Las soluciones fueron preparadas en la escala de molalidad utilizando el método gravimétrico con una incertidumbre de $\pm 3 \times 10^{-4} \mathrm{~mol}_{\text {. }} \mathrm{kg}^{-1}$, en recipientes de vidrio con tapa, tomando todas las precauciones necesarias para evitar la contaminación de las muestras y la pérdida de masa por evaporación de los líquidos utilizados. Todas las medidas de masa fueron realizadas en una balanza analítica Ohaus con una 
incertidumbre de $\pm 1 \times 10^{-4} \mathrm{~g}$. Las densidades de los componentes puros y sus mezclas fueron determinadas en un densímetro de tubo vibratorio Anton Paar DMA 5000, con una incertidumbre de $\pm 1 \times 10^{-5} \mathrm{~g}_{\mathrm{cm}} \mathrm{cm}^{-3} \mathrm{y}$ un control de temperatura de $\pm 0.001 \mathrm{~K}$ desde 283.15 hasta $313.15 \mathrm{~K}$ a intervalos de $5 \mathrm{~K}$ (Instruction Manual Model DMA 5000, Anton Paar, Austria).

\section{RESULTADOS Y DISCUSIÓN}

Los datos experimentales de densidad, de los distintos sistemas seudobinarios derivados del $\left[\mathrm{Bmim}^{+}\right][\mathrm{Cl}]$ fueron ajustados por un procedimiento de mínimos cuadrados usando la ecuación.

$\rho=a+b m+c T+d m^{2}+e T^{2}+f m T+g m^{3}+h T^{3}+i m T^{2}+j m^{2} T$

Donde $\rho$ es la densidad de la solución, $m$ es la molalidad de la DL-Alanina en la mezcla $\left[\mathrm{Bmim}^{+}\right]\left[\mathrm{Cl}^{-}\right]+$agua, $T$ es la temperatura absoluta y las letras minúsculas desde $a$ hasta $j$ son parámetros ajustables. Los resultados de este ajuste se muestran en la tabla 1 y ellos permiten obtener densidades con la desviación estándar especificada en dicha tabla.

Los valores de los volúmenes molares aparentes fueron calculados de los valores de densidad generados a partir de la ecuación (1) mediante la ecuación (2) (Zhen et al., 2005).

$V_{\phi}=\frac{M}{\rho}-\frac{1000\left(\rho-\rho_{0}\right)}{m \rho \rho_{0}}$

Donde $M$ es la masa molar del aminoácido, $m$ es la molalidad de la DL-Alanina, que es definida como las moles de DL-Alanina por kilogramo de solvente (solución acuosa de $\left[\mathrm{Bmim}^{+}\right]\left[\mathrm{Cl}^{-}\right]$), $\rho$ y $\rho_{0}$ son respectivamente la densidad de la solución (DL-Alanina + solución acuosa de [Bmim+][Cl-]) y la densidad del solvente (solución acuosa de $\left.\left[\mathrm{Bmim}^{+}\right][\mathrm{Cl}]\right)$. Finalmente se obtuvieron los volúmenes molares aparentes a dilución infinita $V_{\phi}^{o}$, con ayuda de la correlación $V_{\phi}=f(m)$ usando la ecuación (3) ahí donde el comportamiento es lineal, tómese como ejemplo el sistema ilustrado en la figura 1.

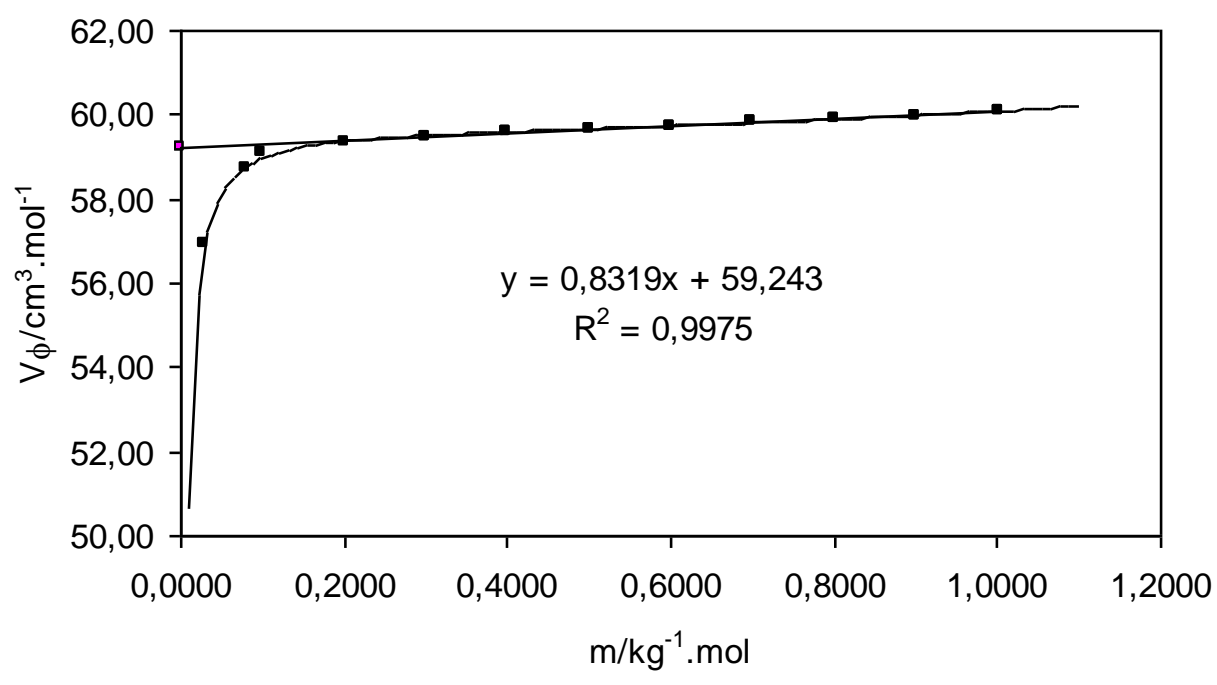

Figura 1. Correlación de $V_{\phi}=f(m)$ para las soluciones de la D-L Alanina en el solvente ([Bmin+][Cl-] + Agua) de concentración $0,0500 \mathrm{~m}$ a $283.15 \mathrm{~K}$, mostrando la región donde se aplica la ecuación (3) que permite determinar el volumen molar aparente a dilución infinita.

$V_{\phi}=V_{\phi}^{o}+S_{v} m$

Donde $S_{v}$ es la pendiente límite experimental. 
Tabla 1. Parámetros de ajuste de la ecuación (1), para las mezclas seudobinarias derivadas de las soluciones acuosas de líquido iónico[Bmim $][$ [Cl-], válida en el intervalo de (0.0000-1.0007) $\mathrm{m}$ (molalidad aminoácido) y a las temperaturas de $(283.15,288.15,293.15,298.15,303.15,308.15$ y 313.15$) \mathrm{K}$.

\begin{tabular}{|c|c|c|c|c|c|c|c|}
\hline$m$ LI $\left(\mathrm{mol}_{\mathrm{Kg}}{ }^{-1}\right)$ & 0,0500 & 0,1000 & 0,2000 & 0,4000 & 0,6000 & 0,8000 & 1,0000 \\
\hline \multicolumn{8}{|c|}{ Parámetros de la ecuación (1) } \\
\hline$a$ & $-0,17661870$ & 0,13744127 & 0,34283875 & 0,08751923 & 0,21196273 & 0,25330157 & 0,14831009 \\
$c$ & 0,12343271 & 0,11759640 & 0,13663855 & 0,14782281 & 0,14119456 & 0,14146428 & 0,16302382 \\
$c$ & 0,01092212 & 0,00779267 & 0,00575147 & 0,00850977 & 0,00739611 & 0,00711739 & 0,00830358 \\
$d$ & $-2,7790 \mathrm{E}-03$ & $-4,2959 \mathrm{E}-03$ & $-4,4956 \mathrm{E}-03$ & $-4,8718 \mathrm{E}-03$ & $-4,7707 \mathrm{E}-03$ & $-4,6003 \mathrm{E}-03$ & $-4,9780 \mathrm{E}-03$ \\
$e$ & $-3,2756 \mathrm{E}-05$ & $-2,2334 \mathrm{E}-05$ & $-1,5496 \mathrm{E}-05$ & $-2,5221 \mathrm{E}-05$ & $-2,1800 \mathrm{E}-05$ & $-2,1176 \mathrm{E}-05$ & $-2,5430 \mathrm{E}-05$ \\
$f$ & $-5,8952 \mathrm{E}-04$ & $-5,4765 \mathrm{E}-04$ & $-6,7637 \mathrm{E}-04$ & $-7,5148 \mathrm{E}-04$ & $-7,0772 \mathrm{E}-04$ & $-7,1074 \mathrm{E}-04$ & $-8,5643 \mathrm{E}-04$ \\
$g$ & $-4,01440 \mathrm{E}-05$ & $5,36973 \mathrm{E}-05$ & $5,79002 \mathrm{E}-05$ & $4,91346 \mathrm{E}-05$ & $2,10231 \mathrm{E}-05$ & $2,08023 \mathrm{E}-05$ & $-1,59750 \mathrm{E}-05$ \\
$h$ & $3,13054 \mathrm{E}-08$ & $1,97272 \mathrm{E}-08$ & $1,20517 \mathrm{E}-08$ & $2,33704 \mathrm{E}-08$ & $1,98216 \mathrm{E}-08$ & $1,93967 \mathrm{E}-08$ & $2,43769 \mathrm{E}-08$ \\
$i$ & $9,11033 \mathrm{E}-07$ & $8,40312 \mathrm{E}-07$ & $1,05766 \mathrm{E}-06$ & $1,18326 \mathrm{E}-06$ & $1,11071 \mathrm{E}-06$ & $1,11726 \mathrm{E}-06$ & $1,36231 \mathrm{E}-06$ \\
$j$ & $2,08354 \mathrm{E}-06$ & $6,14372 \mathrm{E}-06$ & $6,83380 \mathrm{E}-06$ & $8,10778 \mathrm{E}-06$ & $7,87218 \mathrm{E}-06$ & $7,30330 \mathrm{E}-06$ & $8,88068 \mathrm{E}-06$ \\
$\sigma$ & $2,86 \mathrm{E}-05$ & $1,80 \mathrm{E}-05$ & $1,63 \mathrm{E}-05$ & $1,78 \mathrm{E}-05$ & $2,11 \mathrm{E}-05$ & $2,21 \mathrm{E}-05$ & $2,07 \mathrm{E}-05$ \\
\hline
\end{tabular}

Los resultados obtenidos para $V_{\phi}^{o}, S_{v}$ y $R^{2}$ se muestran en la tabla 2. Aquí se observa que el $V_{\phi}^{0}$ disminuye con el aumento de la concentración del cosoluto $\left[\mathrm{Bmim}^{+}\right]\left[\mathrm{Cl}^{-}\right]$a todas las temperaturas, produciendo una contracción del volumen a dilución infinita probablemente debido a que las interacciones soluto-cosoluto aumentan. Adicionalmente también se observa que los valores de $V_{\phi}^{0}$ aumentan con la temperatura, lo cual podría ser debido a la liberación de algunas moléculas de disolvente desde las capas de solvatación del soluto DL-Alanina hacia la solución.

Por otra parte, los valores experimentales de $V_{\phi}^{o}$ fueron correlacionados con temperatura usando la ecuación (4):

$V_{\phi}^{0}=A+B T+C T^{2}$

Donde $A, B$ y $C$ son constantes empíricas y $T$ es la temperatura absoluta. Los valores de $\left(\partial^{2} V_{\phi}^{0} / \partial T^{2}\right)$ encontrados a partir de la ecuación anterior resultaron negativos, tal como se aprecia en la tabla 3.

Esto sugiere de acuerdo con el criterio de Hepler, que la DL-Alanina se comporta como un soluto disruptor de la estructura del solvente en soluciones acuosas de $[\mathrm{Bmim}+][\mathrm{Cl}]$. Se sabe que los factores que contribuyen al comportamiento hidrofílico de la DL-Alanina, podrían ser debidos a la formación de puentes de hidrógeno en la solución asociados a interacciones ión-grupo no polar entre el cosoluto $\left[\mathrm{Bmim}^{+}\right]\left[\mathrm{Cl}^{-}\right]$y el soluto DL-Alanina, es decir, interacciones de las partes apolares de los iones $\left[\mathrm{Bmim}^{+}\right]$del líquido iónico y el grupo $\mathrm{COO}^{-}$del aminoácido o entre los iones $\mathrm{Cl}^{-}$del cosoluto, y el grupo $\mathrm{CH}_{3}$ de la DL-Alanina o a interacciones entre $\mathrm{Cl}^{-}$, del cosoluto y el grupo $\mathrm{NH}_{3}{ }^{+}$del aminoácido (Hepler, 1969., Makhatadze et al., 1992; Páez et al., 2014).

Otra forma de examinar las interacciones en solución tiene que ver con el análisis de los volúmenes molares parciales de transferencia a dilución infinita. Este análisis parte del concepto de que el estado de dilución infinita, se caracteriza por la ausencia de las interacciones soluto-soluto, por tanto los volúmenes molares de transferencia a dilución infinita en nuestro caso, de la DL-Alanina desde el agua pura hasta las soluciones de [Bmim+][Cl] proporcionan información acerca de la interacción soluto-cosoluto (Pal et al., 2011; Páez F., et al., 2014). Los valores de $\Delta V_{\phi}^{o}$ son obtenidos aplicando la ecuación (5) y los resultados se muestran en la tabla 4.

$\Delta V_{\phi}^{o}=V_{\phi}^{o}\left(\left[\mathrm{Bmim}^{+}\right][\mathrm{Cl}]+\right.$ Agua + DL-Alanina $)-V_{\phi}^{o}($ DL-Alanina + Agua $)$

Se puede observar que los valores de $\Delta V_{\phi}^{o}$ disminuyen con el incremento de la concentración de [Bmim $\left.{ }^{+}\right][\mathrm{Cl}]$ en las distintas mezclas. 
Tabla 2: Volumenes molares aparentesa dilución infinita $\boldsymbol{V}_{\boldsymbol{\phi}}^{\boldsymbol{o}}$ y pendiente experimental $\boldsymbol{S}_{\boldsymbol{v}}$ para la DL-Alanina en soluciones acuosas de [Bmim+][Cl] ${ }^{+}$las temperaturas de $(283.15,288.15,293.15,298.15,303.15,308.15$ y 313.15$) \mathrm{K}$.

\begin{tabular}{|c|c|c|c|c|}
\hline$T / K$ & $m\left[\mathrm{Bmim}^{+}\right]\left[\mathrm{Cl}^{-}\right]$ & $V_{\phi}^{o} /\left(\mathrm{cm}^{3} \cdot \mathrm{mol}^{-1}\right)$ & $S_{v} /\left(\mathrm{cm}^{3} \cdot \mathrm{mol}^{-2} \cdot \mathrm{kg}\right)$ & $R^{2}$ \\
\hline \multirow{8}{*}{283.15} & 0.0000 & 59.07 & 12.65 & 0.9987 \\
\hline & 0.0500 & 59.24 & 0.83 & 0.9952 \\
\hline & 0.1000 & 59.16 & 0.83 & 0.9967 \\
\hline & 0.2000 & 59.11 & 0.82 & 0.9988 \\
\hline & 0.4000 & 59.05 & 0.83 & 0.9977 \\
\hline & 0.6000 & 59.00 & 0.83 & 0.9983 \\
\hline & 0.8000 & 58.99 & 0.78 & 0.9997 \\
\hline & 1.0000 & 58.95 & 0.77 & 0.9975 \\
\hline \multirow{8}{*}{288.15} & 0.0000 & 59.62 & 10.38 & 0.9966 \\
\hline & 0.0500 & 59.63 & 0.82 & 0.9992 \\
\hline & 0.1000 & 59.55 & 0.86 & 0.9995 \\
\hline & 0.2000 & 59.50 & 0.87 & 0.9991 \\
\hline & 0.4000 & 59.44 & 0.88 & 0.9985 \\
\hline & 0.6000 & 59.39 & 0.90 & 0.9991 \\
\hline & 0.8000 & 59.36 & 0.89 & 0.9996 \\
\hline & 1.0000 & 59.33 & 0.84 & 0.9991 \\
\hline \multirow{8}{*}{293.15} & 0.0000 & 60.04 & 8.26 & 0.9983 \\
\hline & 0.0500 & 59.95 & 0.83 & 0.9993 \\
\hline & 0.1000 & 59.87 & 0.82 & 0.9994 \\
\hline & 0.2000 & 59.81 & 0.81 & 0.9996 \\
\hline & 0.4000 & 59.77 & 0.82 & 0.9996 \\
\hline & 0.6000 & 59.71 & 0.83 & 0.9980 \\
\hline & 0.8000 & 59.68 & 0.82 & 0.9995 \\
\hline & 1.0000 & 59.65 & 0.78 & 0.9993 \\
\hline \multirow{8}{*}{298.15} & 0.0000 & 60.25 & 9.63 & 0.9962 \\
\hline & 0.0500 & 60.22 & 0.82 & 0.9977 \\
\hline & 0.1000 & 60.11 & 0.78 & 0.9993 \\
\hline & 0.2000 & 60.09 & 0.76 & 0.9990 \\
\hline & 0.4000 & 60.03 & 0.78 & 0.9994 \\
\hline & 0.6000 & 59.98 & 0.75 & 0.9984 \\
\hline & 0.8000 & 59.95 & 0.75 & 0.9997 \\
\hline & 1.0000 & 59.93 & 0.73 & 0.9992 \\
\hline \multirow{8}{*}{303.15} & 0.0000 & 60.56 & 9.11 & 60.568 \\
\hline & 0.0500 & 60.51 & 0.81 & 0.9979 \\
\hline & 0.1000 & 60.43 & 0.75 & 0.9998 \\
\hline & 0.2000 & 60.39 & 0.75 & 0.9990 \\
\hline & 0.4000 & 60.35 & 0.78 & 0.9988 \\
\hline & 0.6000 & 60.29 & 0.77 & 0.9997 \\
\hline & 0.8000 & 60.24 & 0.81 & 0.9994 \\
\hline & 1.0000 & 60.22 & 0.76 & 0.9995 \\
\hline \multirow{8}{*}{308.15} & 0.0000 & 60.92 & 8.91 & 0.9988 \\
\hline & 0.0500 & 60.72 & 0.81 & 0.9963 \\
\hline & 0.1000 & 60.64 & 0.70 & 0.9993 \\
\hline & 0.2000 & 60.59 & 0.71 & 0.9999 \\
\hline & 0.4000 & 60.53 & 0.71 & 0.9994 \\
\hline & 0.6000 & 60.48 & 0.73 & 0.9989 \\
\hline & 0.8000 & 60.45 & 0.73 & 0.9996 \\
\hline & 1.0000 & 60.43 & 0.69 & 0.9985 \\
\hline \multirow{8}{*}{313.15} & 0.0000 & 61.20 & 9.07 & 0.9995 \\
\hline & 0.0500 & 60.86 & 0.84 & 0.9959 \\
\hline & 0.1000 & 60.78 & 0.78 & 0.9983 \\
\hline & 0.2000 & 60.72 & 0.71 & 0.9988 \\
\hline & 0.4000 & 60.67 & 0.69 & 0.9991 \\
\hline & 0.6000 & 60.61 & 0.71 & 0.9994 \\
\hline & 0.8000 & 60.59 & 0.69 & 0.9996 \\
\hline & 1.0000 & 60.56 & 0.57 & 0.9992 \\
\hline
\end{tabular}




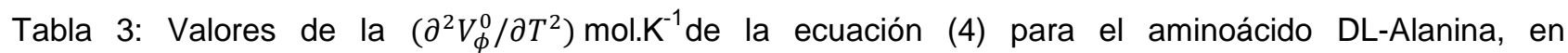
soluciones acuosas de $\left[\mathrm{Bmim}^{+}\right]\left[\mathrm{Cl}^{-}\right]$

\begin{tabular}{cc}
\hline$m\left[\mathrm{Bmim}^{+}\right][\mathrm{Cl}] / \mathrm{mol}_{\mathrm{kg}} \mathrm{kg}^{-1}$ & $\left(\partial^{2} V_{\phi}^{0} / \partial T^{2}\right)$ \\
\hline 0.0500 & $-0.0376 \pm 0.00017$ \\
0.1000 & $-0.0362 \pm 0.00026$ \\
0.2000 & $-0.0380 \pm 0.00002$ \\
0.4000 & $-0.0402 \pm 0.00021$ \\
0.6000 & $-0.0380 \pm 0.00020$ \\
0.8000 & $-0.0360 \pm 0.00016$ \\
1.0000 & $-0.0380 \pm 0.00018$ \\
\hline
\end{tabular}

Tabla 4: Volumenes molares parciales de transferencia a dilución infinita $\Delta \boldsymbol{V}_{\boldsymbol{\phi}}^{\boldsymbol{o}} / \mathrm{cm}^{3} \cdot \mathrm{mol}^{-1}$,para la DL-Alanina en soluciones acuosas de $\left[\mathrm{Bmim}^{+}\right][\mathrm{Cl}]$ a las temperaturas de 283.15, 288.15, 293.15, 298.15, 303.15, 308.15 y $313.15 K$.

\begin{tabular}{cccccccc}
\hline & \multicolumn{7}{c}{$m[\mathrm{Bmim}+][\mathrm{Cl}]\left(\mathrm{mol}_{\mathrm{kg}}-1\right)$} \\
\hline & 0.1000 & 0.2000 & 0.3000 & 0.4000 & 0.5000 & 0.8000 & 1.0000 \\
\hline 283.15 & 0.17 & 0.09 & 0.04 & -0.02 & -0.07 & -0.08 & -0.12 \\
288.15 & 0.01 & -0.07 & -0.12 & -0.18 & -0.23 & -0.26 & -0.29 \\
293.15 & -0.09 & -0.17 & -0.23 & -0.27 & -0.33 & -0.36 & -0.39 \\
298.15 & -0.03 & -0.14 & -0.16 & -0.22 & -0.27 & -0.30 & -0.32 \\
303.15 & -0.05 & -0.13 & -0.17 & -0.22 & -0.27 & -0.32 & -0.34 \\
308.15 & -0.20 & -0.28 & -0.33 & -0.39 & -0.44 & -0.47 & -0.49 \\
313.15 & -0.34 & -0.42 & -0.48 & -0.54 & -0.59 & -0.61 & -0.64 \\
\hline
\end{tabular}

Los volúmenes molares de transferencia $\Delta V_{\phi}^{o}$, de la DL-Alanina desde el agua hacia el solvente seudobinario, resultaron predominantemente negativos y fueron interpretados de manera cualitativa en términos del modelo de solapamiento de coesferas de hidratacion de Gurney (Gurney, 1954) y del criterio de Hepler. Según esta interpretación, en estos sistemas predominan las interacciones entre iones del aminoácido y grupos no polares del cosoluto o visceversa (Mishra y Ahluwalia, 1984) ó más específicamente las siguientes interacciones entre: grupos apolares del $\left[\mathrm{Bmim}^{+}\right]$y el grupo $\mathrm{COO}^{-}$o $\mathrm{NH}_{3}{ }^{+}$del aminoácido; $\mathrm{Cl}^{-}$del cosoluto y el grupo $-\mathrm{CH}_{3}$ de la DL-Alanina; ó interacciones entre $\mathrm{Cl}^{-}$del cosoluto y el grupo $\mathrm{NH}_{3}{ }^{+}$del aminoácido y/o interacciones hidrofóbica-hidrofóbica entre los grupos alquílicos presentes en el líquido iónico y el grupo apolar del aminoácido. De esta manera el predomino de estas interacciones produce un aumento de moléculas de agua liberada desde la esfera de solvatación hacia la fase voluminosa, produciéndose una disminución en la estructura del agua (Liu et al., 2010; Singh et al., 2007); tal como se había anticipado usando el criterio de Hepler.

\section{CONCLUSIONES}

En este estudio, se obtuvieron datos volumétricos para la DL-Alanina en soluciones acuosas de $\left[\mathrm{Bmim}^{+}\right]\left[\mathrm{Cl}^{-}\right]$ a diferentes concentraciones y temperaturas. Los resultados obtenidos para la segunda derivada del $V_{\phi}^{0}$ con respecto a la temperatura indican que la DL-Alanina se comporta como un soluto disruptor de la estructura del solvente en soluciones acuosas de $[\mathrm{Bmim}+[\mathrm{Cl}]$. Adicionalmente, los volúmenes molares aparentes de transferencia a dilución infinita sugieren una disminución de la estructura del agua alrededor de los grupos hidrofóbicos e hidrofílicos. Por otra parte los valores negativos de $\Delta V_{\phi}^{o}$ indican de manera cualitativa que existe un predominio de las interacciones entre los iones del aminoácido y grupos no polares del cosoluto 0 recíprocamente, corroborando con esto lo que se obtuvo al aplicar el criterio de Hepler.

\section{AGRADECIMIENTOS}

Los autores agradecen a la Universidad de Córdoba por el apoyo prestado para la realización de este trabajo. 


\section{REFERENCIAS}

Amani, M., Khodarahmi, R., Ghobadi, S., Mehrabi, M., Kurganov, B. I., Moosavi-Movahedi, A. A., Differential scanning calorimetry study on thermal denaturation of human carbonic anhydrase II, doi: $10.1021 / \mathrm{je} 101087 \mathrm{j}$, J. Chem. Eng. Data 56, 1158-1162 (2011)

Bennion, B. J., Daggett, V., The molecular basis for the chemical denaturation of proteins by urea, doi: 10.1073/pnas.0930122100, Proc. Natl. Acad. Sci. U.S.A., 100(9), 5142-5147 (2003)

Constantinescu, D., Weingärtner, H., Herrmann, C., Protein denaturation by ionic liquids and the hofmeister series: A case study of aqueous solutions of ribonuclease A, doi: 10.1002/anie.200702295, Angew. Chem., 46(46), 8887-8889 (2007)

Dhondge, S. S., Zodape, S. P. y Parwate, D. V., Volumetric and viscometric studies of some drugs in aqueous solutions at different temperatures, doi: 10.1016/j.jct.2011.12.022, The Journal of Chemical Thermodynamics, 48, 207-212 (2012)

England, J. L., Haran, G., Role of solvation effects in protein denaturation: From thermodynamics to single molecules and back, doi: 10.1146/annurev-physchem-032210-103531, Annu. Rev. Phys. Chem., 62, 257-277 (2011)

\section{Gurney, R. W., lonic processes in solutions, McGraw-Hill, New York, USA (1954)}

Hepler, I., Thermal expansion and structure in water and aqueous solutions, http://www.nrcresearchpress.com/doi/pdf/10.1139/v69-762, ISSN: 10.1139/v69-762, Can J. Chem. 47, 46134617 (1969)

Jahagirdhar, D. V., Arbad, B. R., Mirgane, S. R., Lande, M. K., Shankarwar, A. G., Density, ultrasonic velocity and viscosity measurements of four pharmacologically significant drugs in methanol at $25^{\circ} \mathrm{C}$, doi: 10.1016/S0167-7322(97)00021-4, J. Mol. Liq., 75(1), 33-43 (1998)

Liu, C., Volumetric properties of amino acids in aqueous n-methylacetamide solutions at $298.15 \mathrm{~K}$, doi: 10.1007/s10953-010-9585-y, J Solution. Chem., 39, 1253-1263 (2010)

Luciana, I. N. T., Montserrat, D. P., Ana, F. M. C., Mara, G. F., Isabel, M. M., Oscar, C., and João, A. P. C., On the Interactións between Amino Acids and lónic Liquids in Aqueous Media, doi:10.1021/jp906481m, J. Phys. Chem. B, 113, 13971-13979 (2009)

Makhatadze, GI., Privalov, PL., Protein interactions with urea and guanidium chloride: A calorimetric study, www.ncbi.nlm.nih.gov/pubmed/1322462, J. Mol. Biol, 226(2), 491-505 (1992)

Mishra, A. K., Ahluwalia, J. C., Apparent molal volumes of amino acids, $\mathrm{N}$-acetylamino acids, and peptides in aqueous solutions, doi: 10.1021/j150645a021, J. Phys. Chem, 88(1), 86-92 (1984)

Páez, F., Páez, M.S. y Portacio, A., Interacciones de la glicina en soluciones acuosas de tetrafluoroborato de 1-butil, 3-metilimidazolio a diferentes temperaturas, doi:10.5935/0100-4042.20140078, Quim. Nova, 37(3), 418-425 (2014)

Páez, M. S., Miranda, J. A., Torres, F. J., Parámetros de Interacción lónica de las Soluciones Acuosas Concentradas del Líquido lónico Trifluorometanosulfonato de 1-Etil-3-Metil-Imidazolio a Varias Temperaturas, doi: 10.4067/S0718-07642014000600010, Inf. Tecnológica, 25(6), 77-84 (2014)

Páez, M.S., M.K. Vergara y N.A. De la Espriella., Interacciones de la DL-Alanina en soluciones acuosas de trifluorometanosulfonato de 1-butil, 3-metilimidazolio a diferentes temperaturas, doi: 10.4067/S071807642015000100007, Inf. Tecnol., 26(1), 63-70 (2015)

Pal, A. y N. Chauhan, Volumetric behaviour of amino acids and their group contributions in aqueous lactose solutions at different temperatures, doi:10.1016/j.jct.2010.08.004, J. Chem. Thermodynamics: 43, 140-146 (2011)

Qiao, Z., Dang, Y. L. y Zhang, S., Phase equilibrium system of cadmium chloride $+D L$-alanine + water at $298.15 \mathrm{~K}$ and standard molar enthalpy of formation of $\mathrm{Cd}_{3}(\mathrm{Ala})_{2} \mathrm{Cl}_{6} \cdot 4 \mathrm{H}_{2} \mathrm{O}$, doi: $10.1021 / \mathrm{je} 900845 \mathrm{~g}$, J. Chem. Eng., 55(7),2451-2454 (2010) 
Rajagopal, K., Jayabalakrishnan, S. S., Volumetric and viscometric studies of 4-aminobutyric acid in aqueous solutions of salbutamol sulphate at 308.15, 313.15 and $318.15 \mathrm{~K}$, doi:10.1016/S1004-9541(08)60279-7, Chinese Journal of Chemical Engineering, 17(5), 796-804 (2009)

Shekaari, H., Jebali, F., Densities and electrical conductances of amino acids + ionic liquid ([HMIm]Br) $+\mathrm{H} 2 \mathrm{O}$ mixtures at 298.15K, doi:10.1016/j.fluid.2010.04.002, Fluid Phase Equilib, 295(1), 68-75 (2010)

Singh, M., Pandey, M., Yadav, R. K., y Verma, H. S., Thermodynamic studies of molar volume, pair and triplet interactions at increasing side-chain length of $\alpha$-amino acids in aqueous potassium chloride solutions at different concentration and 310.15 K, doi:10.1016/j.molliq.2006.12.029, J. Mol. Liq., 135, 188-191(2007)

Swatloski, R. P., Spear, S. K., Holbrey, J. D., Rogers, R. D. Dissolution of cellose with ionic liquids, doi: 10.1021/ja025790m, J. Am. Chem. Soc., 124,4974-4975 (2002)

van Rantwijk, F., Sheldon, R. A., Biocatalysis in ionic liquids. doi: 10.1021/cr050946x, Chem. Rev., 107(6), 2757-2785 (2007)

Wang, J., Yan, J., Lu, J., Effect of sodium caproate on the volumetric and viscometric properties of glycine, $D L-\alpha$-alanine, and DL- $\alpha$-amino-n-butyric acid in aqueous solutions, doi:10.1016/j.jct.2003.11.001, J. Chem. Thermodynamics, 36, 281-288 (2004)

Yan, Z., Geng, R., Gu, B., Pan, Q., y Wang, J., Densities, electrical conductances, and spectroscopic properties of glycyl dipeptide + ionic liquid $\left(\left[\mathrm{C}_{12} \mathrm{mim}\right] \mathrm{Br}\right)+$ water mixtures at different temperatures, doi:10.1016/j.fluid.2014.01.038, Fluid. Phase.Equilib., 367, 125-134(2014)

Zhen, J., Y. Mei Lu, J Gui., W. Guo Xu y H. Wei Li, Volumetric properties of room temperature ionic liquid 2 The concentrated aqueous solutions of \{1-methyl-3-ethylimidazolium ethyl sulfate + water in a temperature range of $278.2 K$ to $338.2 K$, doi:10.1016/j.jct.2005.03.002, J. Chem. Thermodynamics, 37(11), 1250-1255 (2005) 